\title{
Finite amplitudes helical waves on the surface of the conducting fluid earth core as an example of the self-exciting homopolar heterogeneous dynamo
}

\author{
V. V. Glazkov and O. A. Sinkevich \\ Moscow Power Engineering Institute, 111 250, Krasnokazarmennaya str, 14, Moscow, Russia
}

Received: 9 March 2006 - Revised: 25 October 2006 - Accepted: 25 October 2006 - Published: 1 November 2006

\begin{abstract}
Helical waves of the finite amplitude on a surface of the fluid conducting Earth core are examined for generation of the magnetic field. Estimates are made for the differentially rotating cylindrical conducting fluid body, with the helical surface wave structure on its top. This structure is similar to the self-exciting Faraday-disk homopolar heterogeneous dynamo. Estimations of angular velocity and magnetic field magnitude for a polar vortex on the surface of the Earth's liquid outer core gives reasonable numbers and proves this hypothesis to be of value for further detailed analysis. As magnetic field generation by the helical structure is a surface effect, it is possible to find connection between Earth magnetic field fluctuations and fast relief changes on the iron fluid core - the silicate mantle boundary of the Earth.
\end{abstract}

\section{Introduction}

Roughly the magnetic dynamo schemes can be divided into two main classes: homogenous and heterogeneous ones (Moffat, 1978). To the class of heterogeneous dynamo belong industrial electric dynamo schemes and the self-exciting Faraday-disk homopolar dynamo. The latter was proposed by T. Rikitake and examined by Bullard (1955), see on Fig. 1. It consists of a rotating metal disk with a sliding contact to the wire wound around the rotation axis of the disk. This dynamo is characterized by the presence of insulating spacing between the conducting parts forcing the current in the desired direction. Insulating spacing between functionally different domains of the system and their different topology is the characteristic feature of the heterogeneous dynamo. The magnetic field produces the electromotive force within the certain domain. In the Bullard's dynamo it is a rotating disk. The latter is located away from the domain, where

Correspondence to: O. A. Sinkevich

(oleg.sinkevich@itf.mpei.ac.ru) electric currents generate the axial magnetic field component needed to maintain the electromotive force. As a rule within this domain electric currents flow inside a helical conducting structure. In Bullard's dynamo it is a wire wound around the rotation axis of the disk.

The Bullard's dynamo functions when the direction of the wire wound around the axis coincide with the direction of disk rotation. This coincidence can be interpreted as the positive helicity of the system. As for the electric current and the toroidal magnetic field generated by the dynamo, their direction is defined by direction of the initial seed magnetic field, so the systems with the same helicity can generate magnetic fields of different polarity.

In homogeneous dynamos magnetic fields are generated within singly connected domains with uniform conductivity (as a rule, sphere or cylinder filled with conducting medium are examined). Nowadays, the dynamo effect in homogeneous electrically conducting fluids is widely accepted as the only possible effect to explain the abundance of magnetic fields in the cosmos. (Moffat, 1978; Parker, 1979; Gailitis, 2003). But although this dynamo mechanism is much studied, it is still imperfectly understood, despite recent advances in numerical simulations (Glatzmaier and Roberts, 1995; Stevenson, 2003) and certain successes in experimental verifications (Gailitis, 2003; Stieglitz and Muller, 2001) of some dynamo schemes.

It seems of interest, to find examples of the heterogeneous dynamo in the nature since its mechanical and heat efficiency is much higher than expected efficiency of the homogeneous dynamo. This task looks promising as differentially rotating conducting helical structures are quite often observed. Spiral galaxies are well known, helical waves are probable in the sunspots. In lab experiments, helical waves are observed in Rossby vortexes and on the surface of rotating fluid spheres and cylinders.

Differentially rotating conducting helical structures are also probable on the outer border of the Earth's conducting

Published by Copernicus GmbH on behalf of the European Geosciences Union and the American Geophysical Union. 


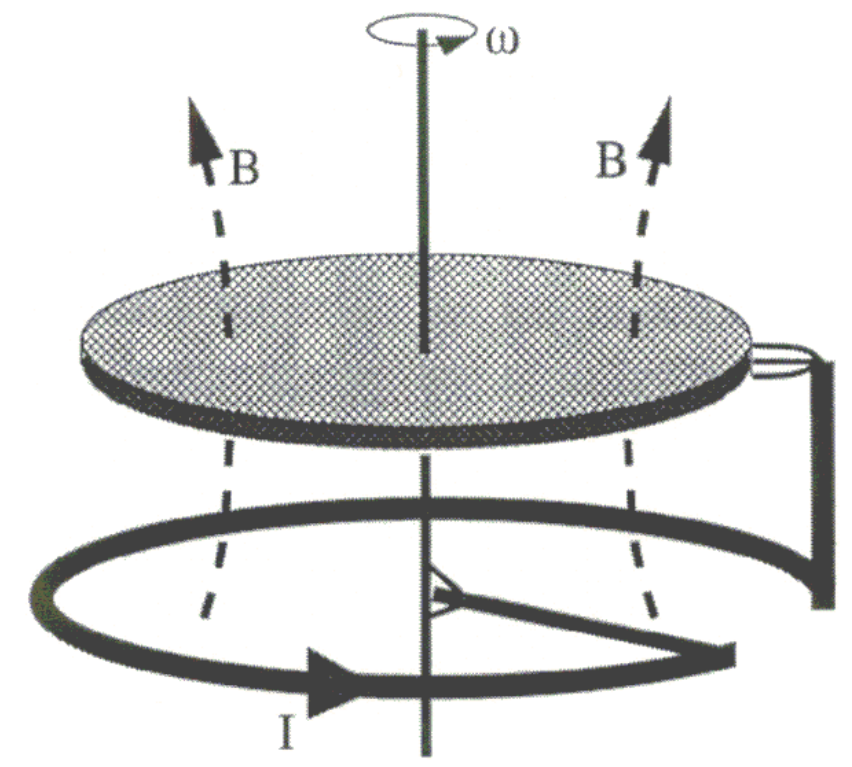

Fig. 1. Bullard's disk dynamo (after Gailitis, 2003).

fluid core, primarily on the poles, as there are some evidences of polar vortexes in the Earth's core (Olson and Aurnou, 1999; Aurnou et al., 2003). It seems that numerical simulations of mantle convection (Glatzmaier and Roberts, 1995) confirm this hypothesis. It is of particular pleasure that the Cowling theorem, 1934, is not correct for helical bodies.

For example in Fig. 2 one can see the experiment where the disk rotates counter-clockwise in the stagnant fluid (Gregory et al., 1955). Disk surface was covered by the special paint. Inside a ring-shaped domain on the disk surface standing vortices in form of logarithm helices were observed. In case of the disk surface being soft the surface had to be deformed in the similar manner. Vortexes appeared due to laminar - turbulent boundary layer transition upon the rigid rotating surface submerged in the stagnant fluid. Transition begins when the Reynolds number $\operatorname{Re}_{1}=r^{2} \Omega / \nu=1.9 \times 10^{5}$ and ends when $R e_{2}=2.8 \times 10^{5}$ (here $v$ - fluid kinematic viscosity). In case of the polar vortex on the core-mantle border, transition to turbulence has to be strongly dumped and transition Reynolds numbers has to be considerably higher as the core surface is not a rigid one.

\section{The estimate of magnetic field generation by the heli- cal structure}

It's worth to begin with simplest estimations of magnetic field generation by the differentially rotating cylinder with surface waves, forming a helical system on its top, Fig. 3. The characteristic length scale of the cylinder is $L$, its external radius and its height are of the same order, rotation rate difference between its top and bottom is $\Omega$. The mean amplitude of helical waves on the top surface is $h$.

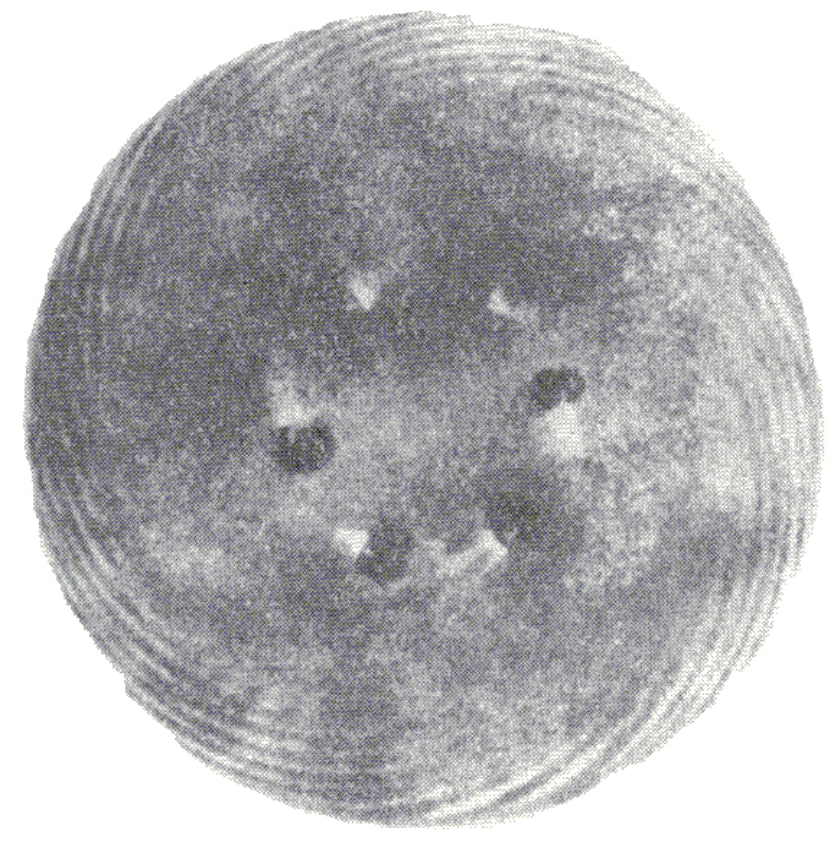

Fig. 2. A disk rotating counter-clockwise in the stagnant fluid (Gregory et al., 1955). The disk surface is covered by the special paint.

If the axial component of magnetic flux density near the cylinder bottom is $B_{z}$, the electromotive force between a rotation axis and a cylinder periphery is of the order $U \sim \Omega L^{2} B_{z}$, while the total electric current inside the cylinder is of the order $I \sim \sigma U L$, where $\sigma$ is the electric conductivity (it is assumed constant) in $\mathrm{S} / \mathrm{m}$. Electric currents inside the cylinder have only radial and axial components everywhere, except in the helical structure on the cylinder top. There, the electric current has also an azimuthal component as it has to flow parallel to the helical surface. The total azimuthal current is of the order $I_{\varphi} \sim I(h / L) \sin (\alpha)$, where $\alpha$ is the helical characteristic angle counted from radial direction. This azimuthal component of currents generates when electric currents flow the along helically shaped surface. The azimuthal component of the current that is located on the cylinder top generates the toroidal magnetic field. Its axial component near the cylinder bottom is of order $B_{z} \sim\left(\mu_{0} / 4 \pi\right) I_{\varphi} / L$, where $\mu_{0}=4 \pi 10^{-7}$ is the permeability of the free space.

For the magnetic field sustained growth, from the initial to the equilibrium value, the emf produced power has to surpass the $\mathrm{Ohm}$ dissipation, so $\mathrm{I} \leq \sigma U L=\sigma \Omega B_{z} L^{3}$. Than, for critical rotation rate $\Omega_{\min }$, we obtain the estimation

$\Omega_{\min } \geq \frac{4 \pi}{\mu_{0}} \frac{1}{\sigma h L \sin (\alpha)}$.

In terms of the "magnetic Reynolds number" $R_{m}=\sigma \mu_{0} \Omega L^{2}$, the first equation looks like

$R_{m}=4 \pi L / h \sin (\alpha)$. 
The magnetic field magnitude can grow until ampere forces do not decrease the system ability to generate the magnetic field. The axial magnetic field interaction with the radial electric current tends to lessen differential rotation of the system. This effect becomes important when ampere forces inside the region of field generation reach the magnitude of inertia forces. By the very rude estimate we obtain $I B L \leq \rho$ $(\Omega L)^{2} L^{2}$ (where $\rho$ - the fluid density). So we get the wellknown limitation on the "Elsasser number" $\Lambda=\sigma B^{2} / 2 \rho \Omega$. Inside the region of field generation, $\Lambda$ has to be less or around a unity:

\section{$\Lambda \leq 1$}

For the expected mean field magnitude in a cylinder we can write then the next estimation for the generated magnetic field,

$B \sim \sqrt{\left(\frac{2 \rho \Omega}{\sigma}\right)}=\sqrt{\frac{8 \pi}{\mu_{0}} \frac{\rho}{\sigma^{2}} \frac{1}{h L \sin \alpha}}$.

For convective vortex on the border of the Earth's fluid conducting outer core with the silicate mantle, we can assume $L=10^{6} \mathrm{~m}$ as the characteristic diameter of the polar vortex (Olson and Aurnou, 1999), $\rho=11 \times 10^{3} \mathrm{~kg} / \mathrm{m}^{3}, \sigma=5 \times 10^{5} \mathrm{~S} / \mathrm{m}$ (Moffat, 1978). As for the surface wave amplitude the value $h=10^{3 \div 4} \mathrm{~m}$ (it is about 0.01 of characteristic polar vortex radius) seems reasonable, though even greater values about $h=10^{5} \mathrm{~m}$ can be expected for developed surface waves, $\sin (\alpha) \sim 0.25$ according to Fig. 2 . Then, we get the polar vortex rotation rate, that is critical for magnetic field generation, about $\Omega \sim 8 \times 10^{-8} \div 8 \times 10^{-9} \mathrm{~s}^{-1}$ or less. It corresponds to fluid motion on the vortex periphery with a velocity $V \sim \Omega L / 2=0.04 \div 0.004 \mathrm{~m} / \mathrm{s}$ or even one order less in case of developed surface waves. The magnetic field estimate for this rotation rate gives a mean flux density $B \sim 10^{-4} \mathrm{~T}$. According to Eq. (2) the magnetic Reynolds number $R_{m}$ is about $10^{3}$, this value corresponds to the estimate of Parker (1979).

The fluid velocity value looks high, too high perhaps. But the effect of the inner fluid core super-rotation with velocities about $V \sim 0.001 \mathrm{~m} / \mathrm{s}$ or more was observed in several numeric simulations and, possibly, and also was found experimental evidence (Glatzmaier and Roberts, 1995; Olson and Aurnou, 1999; Aurnou et al., 2003).

The formulated hypothesis gives some additional background for numerous attempts of theoretical geophysicist to interpret observations of geomagnetic polarity reversals by modeling the phenomenon with the help of hierarchy of selfexciting coupled Faraday-disk homopolar dynamos (Hide and Moroz, 1999).

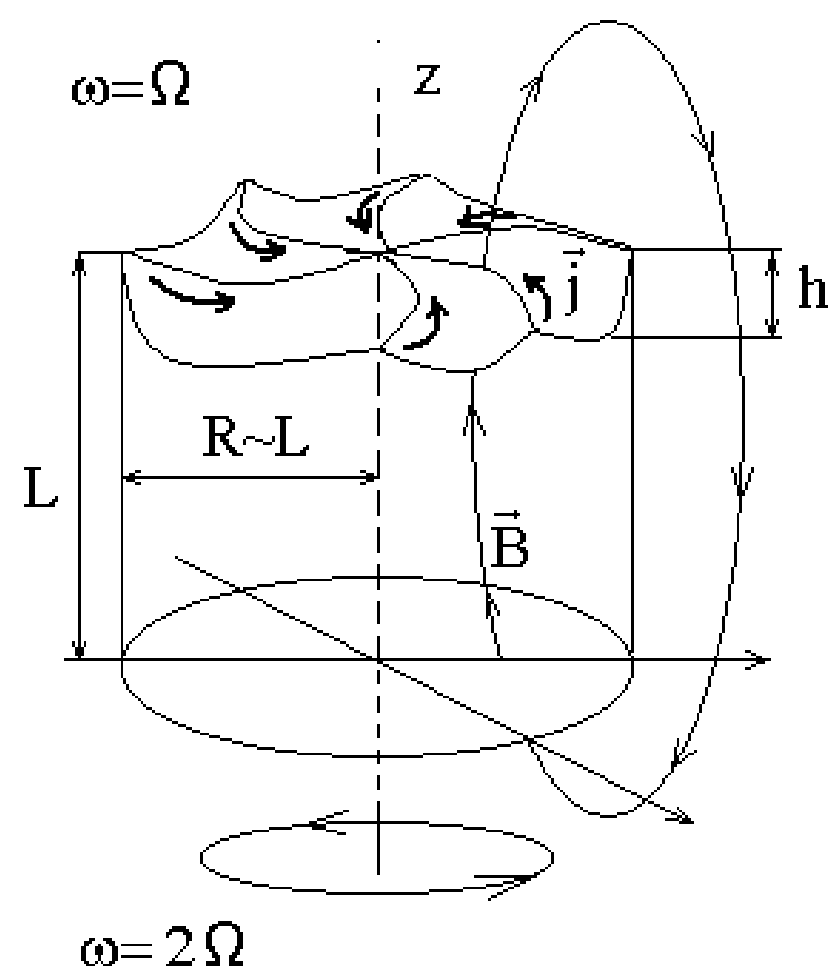

Fig. 3. The differentially rotating conducting cylinder with helical surface waves on its top.

\section{Estimate for self-exciting Faraday-disk homopolar dynamo}

It's worth to examine briefly the self-exciting Faraday-disk homopolar dynamo, similar to differentially rotating cylinder from the Fig. 3. This simplified scheme is depicted in Fig. 4. By accident it resembles to a spiral galaxy with numerous spiral arms.

The radial electric current flows along the rotating disk 1 from the central shaft 2 to the disk periphery. Next, the electric current flows upwards along a cylindrical side surface 3 and reaches the top surface 4 . This top surface is divided into separate helical slices (in Fig. 4 only some of them are shown) by number of helical cuts. The axial magnetic field is generated in this helical structure on the top of a cylinder.

We will assume that this structure has a characteristic height $L$, the rotating disk 1 on its bottom has the characteristic thickness $\delta$, the electric conductivity of the disk material is $\sigma$, the disk external radius $r_{1}=b \cdot L$ and the internal one $r_{2}=a \cdot L$. The rotation rate of the disk is $\Omega$. The total electrical resistance of the disk, for the radial electric current, is equal to

$R_{\mathrm{disk}}=\ln (b / a) /(2 \pi \sigma \delta)$.

The electric resistance of the central shaft 1 , of the side surface 3 of a cylinder and of the top surface 4 are supposed to 


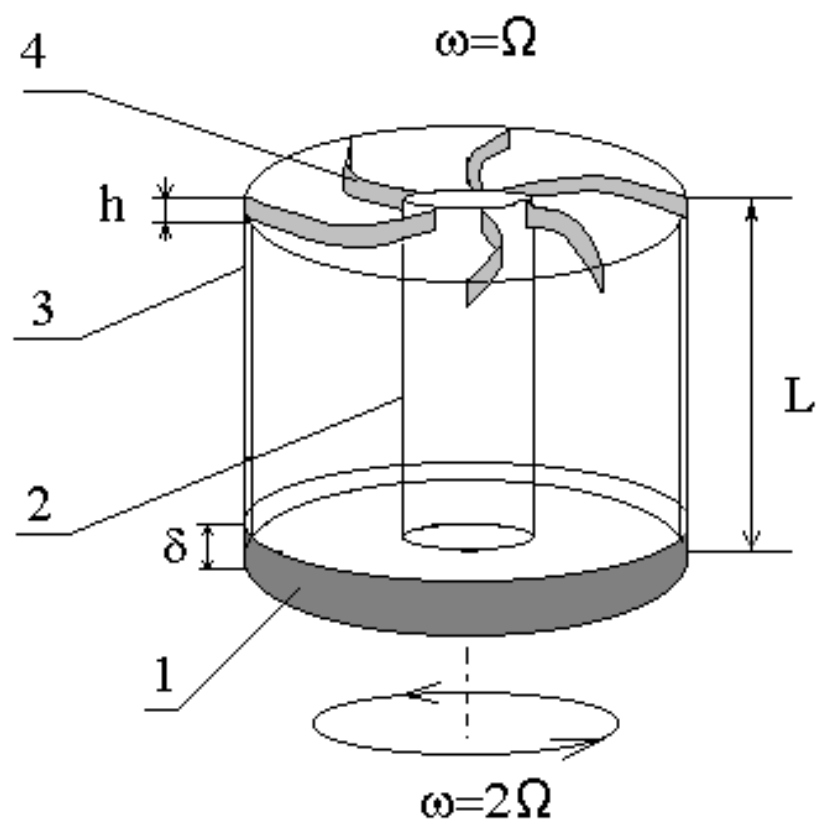

Fig. 4. The self-exciting Faraday-disk homopolar dynamo similar to the differentially rotating conducting cylinder with helical surface waves on its top.

be negligibly small, as they are assumed thick in relation to the disk and their resistance $R_{\text {add }} \sim 1 / \sigma L \ll R_{\text {disk. }}$. The thickness of the top surface 4 is $h, h / L \ll 1$, the number of perforating helical cuts on it is assumed to be very high to simplify integration along the top surface.

In the steady-state dynamo case, the electric current $I$ flowing through the disk 1 satisfy the equation,

$I\left(R_{\mathrm{disk}}+R_{\mathrm{add}}\right)=\int_{r_{2}}^{r_{1}} \Omega r B_{z}(r) d r$.

Here $B_{z}(r)$ is the axial component of magnetic flux density, generated inside the disk by helical currents flowing on the top of the structure 4. Using the Bio-Savart law for magnetic field calculation, and taking into account that only the helical electric current on the top generates the axial magnetic field, the right part of the Eq. (4) looks as,

$$
\begin{aligned}
\int_{r_{2}}^{r_{1}} \Omega r B_{z}(r) d r= & \frac{\mu_{0}}{4 \pi} \varepsilon I \Omega \sin (\alpha) L \int_{a}^{b} \xi d \xi \int_{a}^{b} d \eta \\
& \int_{0}^{2 \pi} \frac{d \phi}{2 \pi} \frac{(\eta-\xi \cos \phi)}{\left(1+\eta^{2}+\xi^{2}-2 \eta \xi \cos \phi\right)^{3 / 2}} .
\end{aligned}
$$

Here $\varepsilon$ is the fraction of the total electric current, which flows through the helical structure on the top. In this case $\varepsilon=1$. The minimal rotation rate $\Omega$ will be:

$\Omega=\frac{4 \pi}{\mu_{0}} \frac{1}{\sigma L \delta \varepsilon \sin (\alpha)} \frac{\ln \left(\frac{b}{a}\right)}{2 \pi Q}$.

The constant $Q$ is defined as

$$
Q=\int_{a}^{b} \xi d \xi \int_{a}^{b} d \eta \int_{0}^{2 \pi} \frac{d \phi}{2 \pi} \frac{(\eta-\xi \cos \phi)}{\left(1+\eta^{2}+\xi^{2}-2 \eta \xi \cos \phi\right)^{3 / 2}} .
$$

In the case of constants $b=1 / 2, a=1 / 8$, the numerical calculation gives $Q \approx 0.01$.

For the lab model made of copper, with $\sigma=5 \times 10^{7} \mathrm{~S} / \mathrm{m}$, $L=1 \mathrm{~m}, \delta=0.05 \mathrm{~m}, \sin (\alpha)=0.5$, the rotation rate for the steady dynamo is equal to $\Omega \sim 150 \mathrm{~s}^{-1}$ and corresponding to the velocity $V \sim 20 \mathrm{~m} / \mathrm{s}$.

The Eq. (5) becomes similar with the Eq. (1) in the case, when the thickness of the rotating disk becomes of order $\delta \sim L$, and through the helical structure on the top flows only some fraction $\varepsilon \sim h / L$ of the total electric current. The latter assumption corresponds to the cylinder being filled with conducting materials.

\section{Conclusions}

Despite a purely illustrative character of above estimates, the following conclusions can be made. The helical conducting structures can generate, at least in some cases, magnetic fields of the considerable magnitude. As differentially rotating conducting helical structures are quite often observed in the nature, this hypothesis seems to be worthy for the further detailed numerical analysis. It literally lays on the surface, as magnetic field generation by helical structures is the "surface effect". If the further analysis confirms these estimates, it can be possible to find connection between Earth magnetic field fluctuations and relatively fast relief changes on the iron fluid core - the silicate mantle boundary of the Earth.

The same approach can be used to examine magnetic field generation process in various celestial bodies (for the sunspots the estimate according Eqs. (1-3) gives quite reasonable numbers).

Acknowledgements. The authors thank Russian Foundation for Basic Research and State Direction of Technical and Scientific Programs for support of this work through the grant 05-08-01217-a and contract 02.445 .11 .7375 .

Edited by: N. S. Erokhin

Reviewed by: G. Cividjian and three other referees

\section{References}

Aurnou, J., Andreadis, S., Zhu, L., and Olson, P.: Experiments on convection in Earth's core tangent cylinder, Earth Planet. Sci. Lett., 212, 119-134, 2003. 
Bullard, E. C.: The Stability of a Homopolar Disc Dynamo, Proc. Camb. Phil. Soc., 51, 744-760, 1955.

Cowling, T. G.: The Magnetic Field of Sunspots, Mon. Not. R. Astr. Soc., 94, 39-48, 1934.

Gailitis, A., Lielausis, O., Platacis, E., Gerbeth, G., and Stefani, F.: The Riga Dynamo Experiment, Surveys in Geophysics, 24, 247-267, 2003.

Glatzmaier, G. A. and Roberts, P. H.: A 3D convective dynamo solution with rotating and finitely conducting inner core and mantle, Phys. Earth Planet Int., 91, 63-75, 1995.

Gregory, N., Stuart, J. T., and Walkker, W. S.: On the stability of three-dimensional boundary layers with application to the flow due to a rotating disk, Phil. Trans. Roy. Soc. Lond., Ser.A, 248, 155-199, 1955.
Hide, R. and Moroz, I. R.: Effects due to induced azimuthal eddy currents in a self-exciting Faraday disk homopolar dynamo with a nonlinear series motor, Physica D, 134, 287-301, 1999.

Moffat, H. K.: Electric Field Generation in Electrically Conducting Fluids, Cambrige University Press, NY, 1978.

Olson, P. and Aurnou, J.: A polar vortex in the Earth's core, Nature, 402, 170-173, 1999.

Parker, E. N.: Cosmical Magnetic Fields: Their Origin and Their Activity, Clarendon Press, Oxford, 1979.

Stevenson, D. J.: Planetary magnetic fields, Earth Planet. Sci. Lett., 208, 1-11, 2003.

Stieglitz, R. and Muller, U.: Experimental Demonstration of a Homogeneous Two-Scale Dynamo, Phys. Fluids, 13, 561-564, 2001. 